\title{
Counting essential surfaces in a closed hyperbolic three-manifold
}

\author{
JEREMY KAHN \\ VLADIMIR MARKOVIĆ
}

Let $\mathbf{M}^{3}$ be a closed hyperbolic three-manifold. We show that the number of genus $g$ surface subgroups of $\pi_{1}\left(\mathbf{M}^{3}\right)$ grows like $g^{2 g}$.

57M50, 20H10

\section{Introduction}

Let $\mathbf{M}^{3}$ be a closed hyperbolic 3-manifold and let $S_{g}$ denote a closed surface of genus $g$. Given a continuous mapping $f: S_{g} \rightarrow \mathbf{M}^{3}$ we let $f_{*}: \pi_{1}\left(S_{g}\right) \rightarrow \pi_{1}\left(\mathbf{M}^{3}\right)$ denote the induced homomorphism.

Definition 1.1 We say that $G<\pi_{1}\left(\mathbf{M}^{3}\right)$ is a surface subgroup of genus $g \geq 2$ if there exists a continuous map $f: S_{g} \rightarrow \mathbf{M}^{3}$ such that the induced homomorphism $f_{*}$ is injective and $f_{*}\left(\pi_{1}\left(S_{g}\right)\right)=G$. Moreover, the subsurface $f\left(S_{g}\right) \subset \mathbf{M}^{3}$ is said to be an essential subsurface.

Recently, we showed [3] that every closed hyperbolic 3-manifold $\mathbf{M}^{3}$ contains an essential subsurface and consequently $\pi_{1}\left(\mathbf{M}^{3}\right)$ contains a surface subgroup. It is therefore natural to consider the question: How many conjugacy classes of surface subgroups of genus $g$ there are in $\pi_{1}\left(\mathbf{M}^{3}\right)$ ? This has already been considered by Masters [5], and our approach to this question builds on our previous work and improves on the work by Masters.

Let $s_{2}\left(\mathbf{M}^{3}, g\right)$ denote the number of conjugacy classes of surface subgroups of genus at most $g$. We say that two surface subgroups $G_{1}$ and $G_{2}$ of $\pi_{1}\left(\mathbf{M}^{3}\right)$ are commensurable if $G_{1} \cap G_{2}$ has a finite index in both $G_{1}$ and $G_{2}$. Let $s_{1}\left(\mathbf{M}^{3}, g\right)$ denote the number surface subgroups of genus at most $g$, modulo the equivalence relation of commensurability. Then clearly $s_{1}\left(\mathbf{M}^{3}, g\right) \leq s_{2}\left(\mathbf{M}^{3}, g\right)$. The main result of this paper is the following theorem. 
Theorem 1.1 Let $\mathbf{M}^{3}$ be a closed hyperbolic 3-manifold. There exist two constants $c_{1}, c_{2}>0$ such that

$$
\left(c_{1} g\right)^{2 g} \leq s_{1}\left(\mathbf{M}^{3}, g\right) \leq s_{2}\left(\mathbf{M}^{3}, g\right) \leq\left(c_{2} g\right)^{2 g},
$$

for $g$ large enough. The constant $c_{2}$ depends only on the injectivity radius of $\mathbf{M}^{3}$.

In fact, Masters shows that

$$
s_{2}\left(g, \mathbf{M}^{3}\right)<g^{c_{2} g}
$$

for some $c_{2} \equiv c_{2}\left(\mathbf{M}^{3}\right)$, and likewise for some $c_{1} \equiv c_{1}\left(\mathbf{M}^{3}\right)$

$$
g^{c_{1} g}<s_{1}\left(g, \mathbf{M}^{3}\right)
$$

when $\mathbf{M}^{3}$ has a self-transverse totally geodesic subsurface. We follow Masters' approach to the upper bound, improving it from $g^{c_{2} g}$ to $\left(c_{2} g\right)^{2 g}$ by more carefully counting the number of suitable triangulations of a genus $g$ surface. Using our previous work [3] we replace Masters' conditional lower bound with an unconditional one, and we improve it from $g^{c g}$ to $\left(c_{1} g\right)^{2 g}$ with the work of Muller and Puchta [6] counting number of maximal surface subgroups of a given surface group. We then make a new subgroup from old in the spirit of Masters' construction, but taking the nearly geodesic subgroup from [3] as our starting point.

The above theorem enables us to determine the order of the number of surface subgroups up to genus $g$. We have the following corollary.

Corollary 1.1 We have

$$
\lim _{g \rightarrow \infty} \frac{\log s_{1}\left(\mathbf{M}^{3}, g\right)}{2 g \log g}=\lim _{g \rightarrow \infty} \frac{\log s_{2}\left(\mathbf{M}^{3}, g\right)}{2 g \log g}=1 .
$$

We make the following conjecture.

Conjecture 1.1 For a given closed hyperbolic 3-manifold $\mathbf{M}^{3}$, there exists a constant $c(M)>0$ such that

$$
\lim _{g \rightarrow \infty} \frac{1}{g} \sqrt[2 g]{s_{i}\left(\mathbf{M}^{3}, g\right)}=c(M), \quad i=1,2 .
$$

Acknowledgements JK was supported by NSF grant DMS 0905812. 


\section{The upper bound}

Fix a closed hyperbolic 3-manifold $\mathbf{M}^{3}$. In this section we prove the upper bound in Theorem 1.1, that is we show

$$
s_{2}\left(\mathbf{M}^{3}, g\right) \leq\left(c_{2} g\right)^{2 g}
$$

for some constant $c_{2}>0$.

\subsection{Genus $g$ triangulations}

We have the following definition.

Definition 2.1 Let $S_{g}$ denote a closed surface of genus $g$. We say that a connected graph $\tau$ is a triangulation of genus $g$ if it can be embedded into the surface $S_{g}$ such that every component of the set $S_{g} \backslash \tau$ is a triangle. The set of genus $g$ triangulations is denoted by $\mathcal{T}(g)$. We say that $\tau \in \mathcal{T}(k, g) \subset \mathcal{T}(g)$ if

- each vertex of $\tau$ has the degree at most $k$,

- the graph $\tau$ has at most $\mathrm{kg}$ vertices and at most $\mathrm{kg}$ edges.

We observe that any given genus $g$ triangulation $\tau$, can be in a unique way (up to a homeomorphism of $S_{g}$ ) be embedded in $S_{g}$.

We say that Riemann surface is $s$-thick is its injectivity radius is bounded below by $s>0$. Every thick Riemann surface has a good triangulation in the sense of the following lemma.

Lemma 2.1 Let $S$ be an $s$-thick Riemann surface of genus $g \geq 2$. Then there exists $k=k(s)>0$ and a triangulation $\tau \in \mathcal{T}(k, g)$ that embeds in $S$, such that every edge of $\tau$ is a geodesic arc of length at most $s$.

Proof Choose a maximal collection of disjoint open balls in $S$ of radius $s / 4$. Let $V$ denote the set of centers of the balls from the collection. We may assume that no four points from $V$ lie on a round circle (we always reduce the radius of the balls by a small amount and move them into a general position). We construct the Delaunay triangulation associated to the set $V$ as follows. We connect two points from $V$ with the shortest geodesic arc between them, providing they belong to the boundary of a closed ball in $S$ that does not contain any other point from $V$. This gives an embedded graph $\tau$. Since no four points from $V$ lie on the same circle the graph $\tau$ is a triangulation. It is elementary to check that $\tau$ has the stated properties, and we leave it to the reader. 
Given any $\pi_{1}$-injective immersion of $g: S_{g} \rightarrow \mathbf{M}^{3}$, we can find a genus $g$ hyperbolic surface $S$, and a map $f: S \rightarrow \mathbf{M}^{3}$ homotopic to $g$, such that $f(S)$ is a pleated surface. Then $f$ does not increase the hyperbolic distance. Let $s$ denote the injectivity radius of $\mathbf{M}^{3}$. It follows that the injectivity radius of $S$ is bounded below by $s$. We choose a triangulation $\tau(S)$ of $S$ that satisfies the conditions in Lemma 2.1.

Let $\mathcal{C}=\left\{C_{1}, \ldots, C_{m}\right\}$ be a finite collection of balls of radius $s / 4$ that covers $\mathbf{M}^{3}$. We may assume that $\mathcal{C}$ is a minimal collection, that is, if we remove a ball from $\mathcal{C}$, the new collection of balls does not cover $\mathbf{M}^{3}$. Let $f_{i}: S_{i} \rightarrow \mathbf{M}^{3}, i=1,2$, be two pleated maps, and denote by $\tau\left(S_{1}\right)$ and $\tau\left(S_{2}\right)$ the corresponding triangulations from Lemma 2.1 of genus $g$ surfaces $S_{1}$ and $S_{2}$. If the genus $g$ triangulations $\tau\left(S_{1}\right)$ and $\tau\left(S_{2}\right)$ are identical, there exists a homeomorphism $h: S_{1} \rightarrow S_{2}$ such that $h\left(\tau\left(S_{1}\right)\right)=\tau\left(S_{2}\right)$. Assume in addition that for every vertex $v$ of $\tau\left(S_{1}\right)$, the points $f_{1}(v)$ and $f_{2}(h(v))$ belong to the same ball $C_{i} \in \mathcal{C}$. Then by [5, Lemma 2.4], the maps $f_{1}$ and $f_{2} \circ h$ are homotopic.

Since the set $\mathcal{C}$ has $m$ elements, there are at most $m$ ways of mapping a given vertex of $\tau$ to the set $\mathcal{C}$. Choose a vertex $v_{1}$ of $\tau$ and choose an image of $v_{1}$ in $\mathcal{C}$, say $v_{1}$ is mapped to $C_{1}$. Let $v_{2}$ be a vertex of $\tau$, such that $v_{2}$ and $v_{1}$ are the endpoints of the same edge.

Each edge of $\tau$ has the length at most $s$, and the balls from $\mathcal{C}$ have the radius $s / 4$. Since $f$ does not increase the distance, and $\mathcal{C}$ is a minimal cover of $\mathbf{M}^{3}$, it follows that $v_{2}$ can be mapped to at most $K$ elements of $\mathcal{C}$, where $K$ is a constant that depends only on $s$. Repeating this analysis to the remaining vertices of $\tau$ yields the estimate

$$
\tilde{s}_{2}\left(\mathbf{M}^{3}, g\right) \leq m K^{k g-1}|\mathcal{T}(k, g)|,
$$

where $\tilde{s}_{2}\left(\mathbf{M}^{3}, g\right)$ denotes the number of conjugacy classes of surface subgroups of genus equal to $g$.

Let $v(k, n)$ denote the set of all graphs on $n$ vertices so that each vertex has the degree at most $k$. Then $|\mathcal{T}(k, g)| \leq|v(k, k g)|$.

Remark Observing the estimate

$$
|v(k, n)| \leq n^{k n},
$$

Masters showed

$$
\tilde{s}_{2}\left(\mathbf{M}^{3}, g\right) \leq g^{D g},
$$

for some constant $D>0$. However, the set $v(k, k g)$ has many more elements than the set $\mathcal{T}(k, g)$. 
The following lemma will be proved in the next subsection.

Lemma 2.2 There exists a constant $C>0$ that depends only on $k$, such that for $g$ large we have

$$
|\mathcal{T}(k, g)| \leq(C g)^{2 g}
$$

Given this lemma we now prove estimate (1). It follows from the Lemma 2.2 that for every $g$ large we have

$$
|\mathcal{T}(k, g)| \leq(C g)^{2 g} .
$$

Combining this with (2) we get that

$$
\tilde{s}_{2}\left(\mathbf{M}^{3}, g\right) \leq m K^{k g-1}(C g)^{2 g} \leq\left(C_{1} g\right)^{2 g}
$$

holds for every $g \geq 2$ for some constant $C_{1}$. Then

$$
\begin{aligned}
s_{2}\left(\mathbf{M}^{3}, g\right) & =\sum_{r=2}^{g} \tilde{s}_{2}\left(\mathbf{M}^{3}, r\right) \\
& =\sum_{r=2}^{g}\left(C_{1} r\right)^{2 r} \\
& \leq\left(c_{2} g\right)^{2 g}
\end{aligned}
$$

for some constant $c_{2}$. This proves the estimate (1).

\subsection{The proof of Lemma 2.2}

Fix a triangulation $\tau \in \mathcal{T}(k, g)$ and denote the set of oriented edges by $E(\tau)$. Let $\mathbb{Q} E(\tau)$ denote the vector space of all formal sums (with rational coefficients) of edges from $E(\tau)$.

Choose a spanning tree $T$ (a spanning tree of a connected graph is a connected tree that contains all of its vertices) for $\tau$. Let $H_{1}\left(S_{g}\right)$ denote the first homology with rational coefficients of the surface $S_{g}$. We define the linear map $\phi: \mathbb{Q} E(\tau) \rightarrow H_{1}\left(S_{g}\right)$ as follows. Let $e \in\left(E(\tau) \backslash T\right.$ ). Then the union $e \cup T$ is homotopic (on $S_{g}$ ) to a unique (up to homotopy) simple closed curve $\gamma_{e} \subset S_{g}$. We let $\phi(e)$ denote the homology class of the curve $\gamma_{e}$ in $H_{1}\left(S_{g}\right)$. We extend the map $\phi$ to $\mathbb{Q} E(\tau)$ by linearity.

Denote the kernel of $\phi$ by $K(\phi)$ and set

$$
H_{1}(\tau, T)=\frac{\mathbb{Q} E(\tau)}{K(\phi)} .
$$


Then the quotient map (also denoted by) $\phi: H_{1}(\tau, T) \rightarrow H_{1}\left(S_{g}\right)$ is injective, and in fact is an isomorphism. Since $\tau$ is a genus $g$ triangulation, the embedding of the triangulation $\tau$ to $S_{g}$ induces the surjective map of the fundamental group of $\tau$ to the fundamental group of $S_{g}$. Then the induced map $\phi$ between the corresponding homology groups is injective.

Let $e_{1}, \ldots, e_{2 g} \in E(\tau)$ denote a set of $2 g$ edges whose equivalence classes generate $H_{1}(\tau, T)$.

Lemma 2.3 Let $X=T \cup\left\{e_{1}, \ldots, e_{2 g}\right\}$. Then every component of the set $S_{g} \backslash X$ is simply connected.

Proof The set $X$ is connected (since it contains the spanning tree $T$, and the tree $T$ contains all the vertices). Suppose that there exists a component of the set $S_{g} \backslash X$ that is not simply connected. Then there exists a simple closed curve $\gamma \subset S_{g}$ that is not homotopic to a point, and such that

$$
\gamma \cap X=\varnothing .
$$

If $\gamma$ is a nonseparating curve then the homology class of $\gamma$ is nontrivial in $H_{1}\left(S_{g}\right)$. Therefore, there exists a nonseparating simple closed $\alpha \subset S_{g}$ that intersects the curve $\gamma$ exactly once. Let $q_{1}, \ldots, q_{2 g} \in \mathbb{Q}$ be such that

$$
\phi\left(q_{1} e_{1}+\cdots+q_{2 g} e_{2 g}\right)=[\alpha],
$$

where $[\alpha] \in H_{1}\left(S_{g}\right)$ denotes the homology class of $\alpha$. Since the intersection pairing between $[\alpha]$ and $[\gamma]$ is nonzero, and $\phi\left(e_{1}\right), \ldots, \phi\left(e_{2 g}\right)$ is a basis for $H_{1}\left(S_{g}\right)$, we conclude that for some $i \in\{1, \ldots, 2 g\}$, the curve $\gamma$ intersects $e_{i} \cup T$, which is a contradiction.

Suppose that $\gamma$ is a separating curve and denote by $A_{1}$ and $A_{2}$ the two components of the set $S_{g} \backslash \gamma$. The set $X$ is connected, and by the assumption it does not intersect $\gamma$. This implies that $X$ is contained in one of the two subsurfaces $A_{i}$, say $X \subset A_{1}$. Then $X \cap A_{2}=\varnothing$.

Since $\gamma$ is not homotopic to a point, each $A_{i}$ is a nonplanar surface with one boundary component. Therefore, the subsurface $A_{2}$ contains a nonseparating simple closed curve $\gamma_{2}$. Then $\gamma_{2}$ is a nonseparating simple closed curve in $S_{g}$ by the above argument we have that $\gamma_{2}$ intersects the set $X$. This is a contradiction since $X \cap A_{2}=\varnothing$.

Let $P_{1}, \ldots, P_{l}$ denote the components of the set $S_{g} \backslash X$. Each $P_{i}$ is a polygon and we let $m_{i}$ denote the number of sides of the polygon $P_{i}$. Since each edge in $X$ can 
appear as a side in at most two such polygons, we have the inequality

$$
\sum_{i=1}^{l} m_{i} \leq 2 k g,
$$

since by definition the triangulation $\tau$ has at most $k g$ edges.

We proceed to prove Lemma 2.2. We can obtain every triangulation $\tau \in \mathcal{T}(k, g)$ as follows. We first choose a spanning tree $T$, which is a tree that has at most $k g$ vertices. Then to the tree $T$ we add $2 g$ edges $e_{1}, \ldots, e_{2 g}$ in an arbitrary way. After adding the edges, at each vertex of the graph $T \cup\left\{e_{1}, \ldots, e_{2 g}\right\}$ we choose a cyclic ordering. We thicken the edges of the graph $T \cup\left\{e_{1}, \ldots, e_{2 g}\right\}$ to obtain the ribbon graph and the corresponding surface $R$ with boundary (if this surface does not have genus $g$ we discard this graph). The boundary components of the surface $R$ are polygonal curves $P_{i}, i=1, . ., l$, made out of the edges from $T \cup\left\{e_{1}, \ldots, e_{2 g}\right\}$. We then choose a triangulation of each polygon $P_{i}$.

It follows from this description that we can bound the number of triangulations from $\mathcal{T}(k, g)$ by $|\mathcal{T}(k, g)| \leq a b c d$, where

$a=$ \{number of unlabeled trees $T$ with $n \leq k g$ vertices $\},$

$b=\left\{\right.$ number of ways of adding $2 g$ unlabeled edges $e_{1}, \ldots, e_{2 g}$ to $\left.T\right\}$,

$c=$ number of cyclic orderings of edges of $\left.T \cup\left\{e_{1}, \ldots, e_{2 g}\right\}\right\}$,

$d=\left\{\right.$ number of triangulations of the polygons $\left.P_{i}\right\}$.

Let $t(n)$ denote the number of different unlabeled trees on $n$ vertices. By [1] we have $t(n) \leq C 12^{n}$, for some universal constant $C>0$. It follows that $a \leq 2 C 12^{\mathrm{kg}}$. The tree $T$ has at most $\mathrm{kg}$ edges, so there are at most $(\mathrm{kg})^{2}$ ways of adding a labeled edge to $T$. All together there are at most $(\mathrm{kg})^{4 g}$ ways of adding a labeled collection of $2 g$ edges to $T$. To obtain the number of ways of adding unlabeled collection of $2 g$ edges we need to divide this number by $(2 g)$ !. This yields the estimate

$$
b \leq \frac{(k g)^{4 g}}{(2 g) !}<\left(k^{2} g\right)^{2 g}
$$

for $g$ large.

Since each vertex of $\tau$ has the degree at most $k$, and $\tau$ has at most $k g$ edges, we obtain the estimate

$$
c \leq(k !)^{k g} .
$$

Let $p(m)$ denote the number of triangulations of a polygon with $m$ sides. Then $p(m)$ is the $(m-2)-$ th Catalan number and we have $p(m)<2^{2 m}$. As above, let $P_{1}, \ldots, P_{l}$ 
denote the polygons that we need to triangulate and let $m_{i}$ denote the number of sides of the polygon $P_{i}$. Then

$$
d \leq \max \prod_{i=1}^{l} p\left(m_{i}\right) \leq 4^{m_{1}+\cdots+m_{l}},
$$

where the maximum is taken over all possible vectors $\left(m_{1}, \ldots, m_{l}\right), 1 \leq l \leq 2 \mathrm{~kg}$, such that $m_{1}+\cdots+m_{l} \leq 2 \mathrm{~kg}$ (see estimate (3) above). But since $m_{1}+\cdots+m_{l} \leq 2 \mathrm{~kg}$ we have $d \leq 4^{2 k g}$.

Putting the estimates for $a, b, c, d$ together we prove the lemma.

Remark If we are given a tree on a surface $S$, along with $2 g$ edges connecting the vertices of the tree (and satisfying the hypothesis of Lemma 2.3) and a map of the resulting graph into $\mathbf{M}^{3}$, the we can determine the map of $S$ into $\mathbf{M}^{3}$, up to homotopy. Thus we need only bound $\left|\mathcal{T}^{\prime}(k, g)\right|$, where $\mathcal{T}^{\prime}(k, g)$ is the set of trees of size at most $k g$, with $2 g$ more edges added; we observe that $\left|\mathcal{T}^{\prime}(k, g)\right|<a b$.

\section{Quasifuchsian representations of surface groups}

\subsection{Generalized pants decomposition and the complex Fenchel-Nielsen coordinates}

For background on complex Fenchel-Nielsen coordinates, see Series [7], Kourouniotis [4], Tan [8] and our previous work [3]. The exposition and notation we use here is in line with [3, Section 2].

Let $X$ be a compact topological surface (possibly with boundary) and let $\rho: \pi_{1}(X) \rightarrow$ $\operatorname{PSL}(2, \mathbb{C})$ be a representation (a homomorphism). We say that $\rho$ is a $K$-quasifuchsian representation if the group $\rho\left(\pi_{1}(X)\right)$ is $K$-quasifuchsian, in which case we can equip $X$ with a complex structure $X=\mathbb{H}^{2} / F$, for some Fuchsian group $F$, such that $f_{*}=\rho \circ \iota$. Here $\iota: F \rightarrow \pi_{1}(X)$ is an isomorphism, and $f_{*}: F \rightarrow f F f^{-1}$ is the conjugation homomorphism, induced by an equivariant $K$-quasiconformal map $f: \partial \mathbb{H}^{3} \rightarrow \partial \mathbb{H}^{3}$.

We will also say that a quasisymmetric map $f: \partial \mathbb{H}^{2} \rightarrow \partial \mathbb{H}^{3}$ is $K$-quasiconformal if it has a $K$-quasiconformal extension to $\partial \mathbb{H}^{3}$.

By $\Pi$ we denote a topological pair of pants with cuffs $C_{i}, i=1,2,3$. Recall that to every representation $\rho: \pi_{1}(\Pi) \rightarrow \operatorname{PSL}(2, \mathbb{C})$, we associate the three half-lengths $\mathbf{h l}\left(C_{i}\right) \in \mathbb{C}_{+} / 2 i \pi \mathbb{Z}$, where $\mathbb{C}_{+}=\{z \in \mathbb{C}: \operatorname{Re}(z)>0\}$. If $\rho$ is quasifuchsian then it 
is uniquely determined by the half-lengths. The conjugacy class $[\rho]$ of a quasifuchsian representation $\rho$ is called a skew pair of pants.

We let $\Pi$ and $\Pi^{\prime}$ denote two pairs of pants and let $\rho: \pi_{1}(\Pi) \rightarrow \operatorname{PSL}(2, \mathbb{C})$ and $\rho^{\prime}: \pi_{1}\left(\Pi^{\prime}\right) \rightarrow \operatorname{PSL}(2, \mathbb{C})$ denote two representations. Suppose that for some $c_{1} \in$ $\pi_{1}(\Pi)$ and $c_{1}^{\prime} \in \pi_{1}\left(\Pi^{\prime}\right)$, that belong to the conjugacy classes of $C_{1}$ and $C_{1}^{\prime}$ respectively, we have $\rho\left(c_{1}\right)=\rho^{\prime}\left(c_{1}^{\prime}\right)$, and $\mathbf{h l}\left(C_{1}\right)=\mathbf{h l}\left(C_{1}^{\prime}\right)$. By $s(C) \in \mathbb{C} /(\mathbf{h l}(C) \mathbb{Z}+2 \pi i \mathbb{Z})$ we denote the reduced twist-bend parameter, which measures how the two skew pairs of pants $[\rho]$ and $\left[\rho^{\prime}\right]$ align together along the axis of the loxodromic transformation $\rho\left(c_{1}\right)=\rho^{\prime}\left(c_{1}^{\prime}\right)$.

A pair $(\tilde{\Pi}, \chi)$ is a generalized pair of pants if $\tilde{\Pi}$ is a compact surface with boundary and $\chi$ is a finite degree covering map $\chi: \widetilde{\Pi} \rightarrow \Pi$, where $\Pi$ is a pair of pants. (We will also call $\widetilde{\Pi}$ a generalized pair of pants if $\chi$ is understood.) By $\chi_{*}: \pi_{1}(\widetilde{\Pi}) \rightarrow \pi_{1}(\Pi)$ we denote an induced homomorphism.

Definition 3.1 Let $(\widetilde{\Pi}, \chi)$ be a generalized pair of pants and

$$
\tilde{\rho}: \pi_{1}(\tilde{\Pi}) \rightarrow \operatorname{PSL}(2, \mathbb{C})
$$

be a representation. We say that $\tilde{\rho}$ is admissible with respect to $\chi$ if it factors through $\chi_{*}$, that is, there exists $\rho: \pi_{1}(\Pi) \rightarrow \operatorname{PSL}(2, \mathbb{C})$ such that $\tilde{\rho}=\rho \circ \chi_{*}$.

Let $\widetilde{C}_{j}, j=1, \ldots, k$, denote the cuffs (the boundary curves) of the surface $\widetilde{\Pi}$, and let $C_{1}, C_{2}, C_{3}$ continue to denote the cuffs of $\Pi$. Then $\chi$ maps each $\widetilde{C}_{j}$ onto some $C_{i}$ with some degree $m_{j} \in \mathbb{N}$. We say that such a curve $\widetilde{C}_{j}$ is a degree $m_{j}$ curve. For every admissible $\tilde{\rho}$ we define the half-length $\mathbf{h l}\left(\widetilde{C}_{j}\right)$ as $\mathbf{h l}\left(\widetilde{C}_{j}\right)=\mathbf{h l}\left(C_{i}\right)$. Let $\widetilde{c_{j}} \in \pi_{1}\left(\widetilde{\Pi}^{0}\right)$ be in the conjugacy class that corresponds to the cuff $\widetilde{C}_{j}$. Then

$$
\mathbf{l}\left(\tilde{\rho}\left(c_{i}\right)\right)=2 m_{j} \mathbf{h l}\left(C_{i}\right)(\bmod (2 \pi i \mathbb{Z})) .
$$

Let $S$ be an oriented closed topological surface with a generalized pants decomposition. By this we mean that we are given a collection $\mathcal{C}$ of disjoint simple closed curves on $S$, such that for every component $\tilde{\Pi}$ of $S \backslash \mathcal{C}$ there is an associated finite cover $\chi: \widetilde{\Pi} \rightarrow \Pi$. Let

$$
\tilde{\rho}: \pi_{1}(S) \rightarrow \operatorname{PSL}(2, \mathbb{C})
$$

be a representation. We make the following assumptions on $\rho$ :

(1) Given a curve $C \in \mathcal{C}$ there exists two (not necessarily different) generalized pairs of pants $\widetilde{\Pi}_{1}$ and $\widetilde{\Pi}_{2}$ that both contain $C$ as a cuff, and that lie on different sides of $C$. Let $\chi_{1}: \widetilde{\Pi}_{1} \rightarrow \Pi_{1}$ and $\chi_{2}: \widetilde{\Pi}_{2} \rightarrow \Pi_{2}$ be the corresponding finite covers, where $\Pi_{1}$ and $\Pi_{2}$ are two pairs of pants. We assume that the restrictions of $\chi_{1}$ and $\chi_{2}$ on the curve $C$ are of the same degree. 
(2) For every generalized pair of pants $\widetilde{\Pi}$ from the above decomposition of $S$, the restriction $\rho: \pi_{1}(\widetilde{\Pi}) \rightarrow \operatorname{PSL}(2, \mathbb{C})$ is admissible with respect to the covering map $\chi: \widetilde{\Pi} \rightarrow \Pi$ (in the sense of Definition 3.1).

(3) For every $C \in \mathcal{C}$, the half-lengths of $C$ coming from the representations $\rho: \pi_{1}\left(\widetilde{\Pi}_{1}\right) \rightarrow \operatorname{PSL}(2, \mathbb{C})$ and $\rho: \pi_{1}\left(\widetilde{\Pi}_{2}\right) \rightarrow \operatorname{PSL}(2, \mathbb{C})$ are one and the same.

Continuing with the above notation, let $C_{i} \subset \Pi_{i}$ denote the cuff such that $\chi_{i}(C)=C_{i}$. Let $\rho_{i}: \pi_{1}\left(\Pi_{i}\right) \rightarrow \operatorname{PSL}(2, \mathbb{C}), i=1,2$, be the representations such that the restriction of $\rho$ to $\pi_{1}\left(\widetilde{\Pi}_{i}\right)$ is equal to $\rho_{i} \circ\left(\chi_{i}\right)_{*}$. We define the reduced twist bend parameter $s(C)$ associated to $\rho$ to be equal to the reduced twist-bend parameter for the representations $\rho_{1}$ and $\rho_{2}$.

So given a closed surface $S$ with a generalized pants decomposition $\mathcal{C}$, and a representation $\rho: \pi_{1}(S) \rightarrow \operatorname{PSL}(2, \mathbb{C})$, we have defined the parameters $\mathbf{h l}(C) \in \mathbb{C}_{+} / 2 k \pi \mathbb{Z}$ and $s(C) \in \mathbb{C} /(\mathbf{h l}(C) \mathbb{Z}+2 \pi i \mathbb{Z})$. The collection of pairs $(\mathbf{h l}(C), s(C)), C \in \mathcal{C}$, is called the reduced Fenchel-Nielsen coordinates. We observe that a representation $\rho: \pi_{1}(S) \rightarrow \operatorname{PSL}(2, \mathbb{C})$ is Fuchsian if and only if all the coordinates $(\mathbf{h l}(C), s(C))$ are real. This is well known (see [3]) when $\mathcal{C}$ is a pants decomposition. The same is true when $\mathcal{C}$ is a generalized pants decomposition. This follows directly from Definition 3.1 and the above three conditions we impose on $\rho$.

The following elementary proposition (see [3]) states that although a representation $\rho: \pi_{1}(S) \rightarrow \operatorname{PSL}(2, \mathbb{C})$ is not uniquely determined by its reduced Fenchel-Nielsen coordinates, it can be in a unique way embedded in a holomorphic family of representations (uniquely means that there is a unique holomorphic family of representations such that $\rho$ can embedded in this family as described in the following lemma).

Proposition 3.1 Fix a closed topological surface $S$ with a generalized pants decomposition $\mathcal{C}$. Let $z \in \mathbb{C}_{+}^{\mathcal{C}}$ and $w \in \mathbb{C}^{\mathcal{C}}$ denote complex parameters. Then there exists a holomorphic (in $(z, w))$ family of representations

$$
\rho_{z, w}: \pi_{1}(S) \rightarrow \operatorname{PSL}(2, \mathbb{C}),
$$

such that $\mathbf{h l}(C)=z(C)(\bmod 2 \pi i \mathbb{Z})$ and $s(C)=w(C)(\bmod \mathbf{h l}(C) \mathbb{Z}+2 \pi i \mathbb{Z})$. Moreover, for any $\left(z_{0}, w_{0}\right) \in \mathbb{C}_{+}^{\mathcal{C}} \times \mathbb{C}^{\mathcal{C}}$, the family of representations $\rho_{z, w}$ is uniquely determined by the representation $\rho_{z_{0}}, w_{0}$.

The representation $\rho_{z, w}$ is Fuchsian if and only if both $z$ and $w$ are real, that is $z \in \mathbb{R}_{+}^{\mathcal{C}}$ and $w \in \mathbb{R}^{\mathcal{C}}$. In this case the group $\rho_{z, w}\left(\pi_{1}(S)\right)$ is of course discrete. Moreover, in [4] it has been proved that all quasifuchsian representations (up to conjugation in $\operatorname{PSL}(2, \mathbb{C}))$ of $\pi_{1}(S)$ correspond to some neighborhood of the set $\mathbb{R}_{+}^{\mathcal{C}}$ and $\mathbb{R}^{\mathcal{C}}$. But in 
general, little is known for which choice of parameters $z, w$ the group $\rho_{z, w}\left(\pi_{1}(S)\right)$ will be discrete. In the next subsection we prove the following result in this direction. Start with a nearly Fuchsian group $G<\operatorname{PSL}(2, \mathbb{C})$. We obtain a new group $G_{1}<\operatorname{PSL}(2, \mathbb{C})$ from $G$ by bending (by some definite angles) along some sparse equivariant collection of geodesics whose endpoints are in the limit set of $G$. Then the new group $G_{1}$ is also quasifuchsian (although it is not nearly Fuchsian anymore).

\subsection{Small deformations of a sparsely bent pleated surface}

We let $S$ continue to denote a closed surface with a generalized pants decomposition $\mathcal{C}$, and we fix a holomorphic family of representations $\rho_{z, w}$ as in Proposition 3.1. We set $G(z, w)=\rho_{z, w}\left(\pi_{1}(S)\right)$.

Let $\mathcal{C}_{0} \subset \mathcal{C}$ denote a subcollection of curves. For $z \in \mathbb{R}_{+}^{\mathcal{C}}$ and $w \in \mathbb{R}^{\mathcal{C}}$, we let $S_{z, w}$ denote the Riemann surface isomorphic to $\mathbb{H}^{2} / G(z, w)$, and on $S_{z, w}$ we identify the curves from $\mathcal{C}$ with the corresponding geodesics representatives. By $\mathcal{K}\left(S_{z, w}\right)$ we denote the largest number so that the collection of collars (of width $\mathcal{K}\left(S_{z, w}\right)$ ) around the curves from $\mathcal{C}_{0}$ is disjoint on $S_{z, w}$. For each $C \in \mathcal{C}_{0}$, we choose a number $-(3 / 4) \pi<\theta_{C}<(3 / 4) \pi$ (for each curve $C \in\left(\mathcal{C} \backslash \mathcal{C}_{0}\right)$ we set $\left.\theta_{C}=0\right)$.

The purpose of this subsection is to prove the following theorem.

Theorem 3.1 There exist constants $K>1$ and $D>0$ such that the following holds. Let $z_{0} \in \mathbb{R}_{+}^{\mathcal{C}}$ and $w_{0} \in \mathbb{R}^{\mathcal{C}}$, and $z_{1} \in \mathbb{C}_{+}^{\mathcal{C}}$ and $w_{1} \in \mathbb{C}^{\mathcal{C}}$ be such that the representation $\rho=\rho_{z_{1}, w_{1}} \circ \rho_{z_{0}, w_{0}}^{-1}: G\left(z_{0}, w_{0}\right) \rightarrow G\left(z_{1}, w_{1}\right)$, is $K$-quasifuchsian. Set $z_{2}=z_{1}$ and $w_{2}=w_{1}+i \theta_{C}$. If $\mathcal{K}\left(S_{z_{0}, w_{0}}\right) \geq D$, then the representation $\rho_{z_{2}, w_{2}}: \pi_{1}(S) \rightarrow \operatorname{PSL}(2, \mathbb{C})$ is $K_{1}$-quasifuchsian, where $K_{1}$ depends only on $K$ and $D$.

The following lemma is elementary.

Lemma 3.1 Let $0 \leq \theta_{0}<\pi$ and $B_{0} \geq 1$. There exist constants $L\left(\theta_{0}, B_{0}\right)>0$ and $D\left(\theta_{0}, B_{0}\right)>0$ such that the following holds. Let $I \subset \mathbb{R}$ be an interval that is partitioned into intervals $I_{j}, j=1, \ldots, k$. Let $\psi: I \rightarrow \mathbb{H}^{3}$ be a continuous map, such that $\psi$ maps each $I_{j}$ onto a geodesic segment and the restriction of $\psi$ on $I_{j}$ is $B_{0}$-bilipschitz. Assume in addition that the bending angle between two consecutive geodesic intervals $\psi\left(I_{j}\right)$ and $\psi\left(I_{j+1}\right)$ is at most $\theta_{0}$. If the length of every $I_{j}$ is at least $D\left(\theta_{0}, B_{0}\right)$ then $\psi$ is $L\left(\theta_{0}, B_{0}\right)$-bilipschitz.

Let $\psi: I \rightarrow \mathbb{H}^{3}$ be a $C^{1}$ map, where $I \subset \mathbb{R}$ is a closed interval. For $x \in I$ let $v(x) \in T^{1} I$ denote the unit vector that points toward $+\infty$. Let $\delta>0$. We say that the 
map $\psi$ is $\delta$-nearly geodesic if for every $x, y \in I$ such that $x<y \leq x+1$, we have that the angle between the vector $\psi_{*}(v(x))$ and the oriented geodesic segment from $\psi(x)$ to $\psi(y)$ is at most $\delta$.

Clearly, every 0-nearly geodesic map is an isometry, and a sequence of normalized $\delta_{n}$-nearly geodesic maps converges (uniformly on compact sets) in the $C^{1}$ sense to an isometry, when $\delta_{n} \rightarrow 0$. The following lemma is a generalization of the previous one.

Lemma 3.2 There exist universal constants $L, D, \delta>0$, such that the following holds. Suppose that $I$ is partitioned into intervals $I_{j}, j=1, \ldots, k$, and let $\psi: I \rightarrow \mathbb{H}^{3}$ be a continuous map, whose restriction on every closed subinterval $I_{j}$ is $C^{1}$ and $\delta$-nearly geodesic. Assume that the bending angle between two consecutive curves $\psi\left(I_{j}\right)$ and $\psi\left(I_{j+1}\right)$ is at most $3 \pi / 4$ (by the bending angle between two $C^{1}$ curves we mean the appropriate angle determined by the two tangent vectors at the point where the two curves meet). If the length of every $I_{j}$ is at least $D$ then $\psi$ is $L$-bilipschitz.

Proof Choose any two numbers $3 \pi / 4<\theta_{0}<\pi$ and $B_{0}>1$. Assuming that $D>$ $D\left(\theta_{0}, B_{0}\right)$ we can partition each $I_{j}$ into subintervals of length between $D\left(\theta_{0}, B_{0}\right)$ and $2 D\left(\theta_{0}, B_{0}\right)$. Replacing each $I_{j}$ with these new intervals we obtain the new partition of $I$ into intervals $J_{i}$, where each $J_{i}$ has the length between $D\left(\theta_{0}, B_{0}\right)$ and $2 D\left(\theta_{0}, B_{0}\right)$. Let $\phi: I \rightarrow \mathbb{H}^{3}$ be the continuous map that agrees with $\psi$ at the endpoints of all intervals $J_{i}$, and such that the restriction of $\phi$ to each $J_{i}$ maps $J_{i}$ onto a geodesic segment in $\mathbb{H}^{3}$, and is affine (the map $\phi$ either stretches or contracts distances by a constant factor on a given $J_{i}$ ).

Next, since we have the upper bound $2 D\left(\theta_{0}, B_{0}\right)$ on the length of each interval $J_{i}$, we can choose $\delta>0$ small enough such that the bending angle between two consecutive geodesic segments $\phi\left(J_{i}\right)$ and $\phi\left(J_{i+1}\right)$ is at most $\theta_{0}$. Also, by choosing $\delta$ small we can arrange that the map $\phi \circ \psi^{-1}$ is 2 -bilipschitz (the same statement holds if we replace 2 by any other number greater than 1$)$. By the previous lemma the map $\phi$ is $L\left(\theta_{0}, B_{0}\right)-$ bilipschitz. Then the map $\psi$ is $2 L\left(\theta_{0}, B_{0}\right)$-bilipschitz. We take $L=2 L\left(\theta_{0}, B_{0}\right)$, and $D=2 D\left(\theta_{0}, B_{0}\right)$, and the lemma is proved.

We are now ready to prove Theorem 3.1.

Proof Recall that $f: \partial \mathbb{H}^{2} \rightarrow \partial \mathbb{H}^{3}$ is a $K$-quasiconformal map that conjugates $G\left(z_{0}, w_{0}\right)$ to $G\left(z_{1}, w_{1}\right)$. Let $\tilde{f}: \mathbb{H}^{2} \rightarrow \mathbb{H}^{3}$ denote the Douady-Earle extension of $f$.

Remark Usually the Douady-Earle extension refers to the barycentric extensions of a homeomorphism $f: \partial S^{1} \rightarrow \partial S^{1}$ (see [2]). In the same paper (see [2, Section 11]) 
Douady and Earle have shown that in a similar vein one defines the barycentric extension of any homeomorphism $f: \partial S^{k} \rightarrow \partial S^{k}$, for any $k>0$. Similarly one can define the barycentric extension $\widetilde{f}: \mathbb{H}^{m} \rightarrow \mathbb{H}^{n}$ of any continuous map $f: \partial S^{m-1} \rightarrow \partial S^{n-1}$. Alternatively, given a K-quasiconformal map $f: \partial \mathbb{H}^{2} \rightarrow \partial \mathbb{H}^{3}$ we first extend $f$ to an equivariant $K$-quasiconformal map $\bar{f}: \partial \mathbb{H}^{3} \rightarrow \partial \mathbb{H}^{3}$ and then take the corresponding Douady-Earle extension of $\tilde{f}: \mathbb{H}^{3} \rightarrow \mathbb{H}^{3}$ of $\bar{f}$ (as defined by Douady and Earle [2]). The restriction of $\tilde{f}$ to $\mathbb{H}^{2}$ is also called $\tilde{f}$.

Then $\tilde{f}$ is $\delta$-nearly geodesic (this means that the restriction of $\tilde{f}$ to every geodesic segment is $\delta$-nearly geodesic in the sense of the above definition) for some $\delta=\delta(K)$, and $\delta(K) \rightarrow 0$, when $K \rightarrow 1$.

If we assume that $\mathcal{K}\left(S_{z_{0}}, w_{0}\right)$ is large enough, by adjusting $\tilde{f}$, we can arrange that $\tilde{f}$ is then $C^{\infty}$ mapping that maps the geodesics in $\mathbb{H}^{2}$ that are lifts of the geodesics from $\mathcal{C}_{0}$ onto the corresponding geodesics in $\mathbb{H}^{3}$, and ensure that $\tilde{f}$ is $2 \delta$-nearly geodesic. Moreover, we can arrange that $\tilde{f}$ is conformal at every point of every geodesic $\gamma$ that is a lift of a curve from $\mathcal{C}_{0}$.

We construct the map $\tilde{g}: \mathbb{H}^{2} \rightarrow \mathbb{H}^{3}$ that conjugates $G\left(z_{0}, w_{0}\right)$ to $G\left(z_{2}, w_{2}\right)$ as follows.

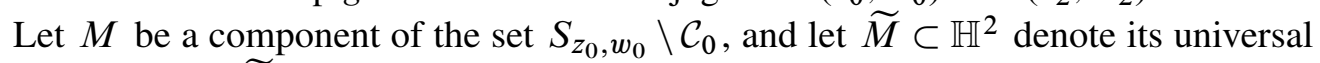
cover, that is, $\widetilde{M}$ is an ideal polygon with infinitely many sides in $\mathbb{H}^{2}$, whose sides are lifts of the geodesics from $\mathcal{C}_{0}$ that bound $M$. We set $\widetilde{g}=\widetilde{f}$ on $\widetilde{M}$.

Let $\widetilde{M}_{1} \subset \mathbb{H}^{2}$ be the universal cover of some other component $M_{1}$ of the set $S_{z_{0}, w_{0}} \backslash \mathcal{C}_{0}$. Let $\gamma$ denote a lift of a geodesic $C \in \mathcal{C}_{0}$, and assume that the polygons $\widetilde{M}$ and $\widetilde{M}_{1}$ are glued to each other along $\gamma$ (that is, $C$ is in the boundary of both $M$ and $M_{1}$ ). Let $R\left(\theta_{C}\right) \in \operatorname{PSL}(2, \mathbb{C})$, denote the rotation about $\tilde{g}(\gamma)$ for the angle $\theta_{C}$. We define $\tilde{g}$ on $\widetilde{M}_{1}$ by letting $\widetilde{g}=R\left(\theta_{C}\right) \circ \widetilde{f}$. We then define $\widetilde{g}$ inductively on the rest of $\mathbb{H}^{2}$.

Clearly $\tilde{g}$ conjugates $G\left(z_{0}, w_{0}\right)$ to $G\left(z_{2}, w_{2}\right)$. Let $x \in \gamma$, and $v(x)$ a nonzero vector that is orthogonal to $\gamma$. Since $\left|\theta_{C}\right| \leq(3 / 4) \pi$, and since $\tilde{f}$ is differentiable at $x$, it follows that the bending angle between the vectors $\tilde{g}_{*}(v(x))$ and $\tilde{g}_{*}(-v(x))$ is at most $(3 / 4) \pi$. If $u(x)$ is any other vector at $x$, since $\widetilde{f}$ is conformal at $x$, it follows that the bending angle between the vectors $\tilde{g}_{*}(u(x))$ and $\tilde{g}_{*}(-u(x))$ is at most as big as the bending angle between the vectors $\tilde{g}_{*}(v(x))$ and $\tilde{g}_{*}(-v(x))$. Therefore, the restriction of the map $\tilde{g}$ on every geodesic segment satisfies the assumptions of Lemma 3.2. It follows that $\hat{g}$ is $L$-bilipschitz, where $L$ depends only on $K$ and $D$. Therefore the representation $\rho_{z_{2}, w_{2}}: \pi_{1}(S) \rightarrow \operatorname{PSL}(2, \mathbb{C})$ is $K_{1}$-quasifuchsian, where $K_{1}$ depends only on $K$ and $D$. 


\subsection{Convex hulls and pleated surfaces}

In this subsection we digress from the notions of generalized pants decompositions and Fenchel-Nielsen coordinates, to prove a preliminary lemma about hyperbolic convex hulls of quasicircles.

Let $\lambda$ be a discrete geodesic lamination in $\mathbb{H}^{2}$, and let $\mathcal{K}(\lambda)$ denote the largest number such that for every small $\epsilon>0$, the collection of collars (crescent in $\mathbb{H}^{2}$ ) of width $\mathcal{K}(\lambda)-\epsilon$ around the leafs of $\lambda$ is disjoint in $\mathbb{H}^{2}$. Let $\mu$ denote a real valued measure on $\lambda$. By $\iota_{\lambda, \mu}=\iota: \mathbb{H}^{2} \rightarrow \mathbb{H}^{3}$, we denote the corresponding pleating map. As usual, by $\iota(\lambda)$ we denote the collection of geodesics in $\mathbb{H}^{3}$ that are images of geodesics from $\lambda$ under $\iota$. If the map $\iota$ is $L$-bilipschitz then $\iota$ extends continuously to a $K-$ quasiconformal map $f: \partial \mathbb{H}^{2} \rightarrow \partial \mathbb{H}^{3}$, for some $K=K(L)$. In this case, let $W \subset \mathbb{H}^{3}$ denote the convex hull of the quasicircle $\iota\left(\partial \mathbb{H}^{2}\right)$. The convex hull $W$ has two boundary components which we denote by $\partial_{1} W$ and $\partial_{2} W$. We prove the following lemma.

Lemma 3.3 There exist universal constants $C_{1}, \delta_{1}>0$, with the following properties. Assume that $\mathcal{K}(\lambda)>C_{1}$, and that $\pi / 4 \leq|\mu(l)| \leq 3 \pi / 4$, for every $l \in \lambda$. Then for every geodesic $\gamma \subset W$ the following holds:

(1) If $\gamma \in \iota(\lambda)$, then for every point $p \in \gamma$, the inequality

$$
\max _{i=1,2} d\left(p, \partial_{i} W\right)>\delta_{1}
$$

holds.

(2) If $\gamma$ does not belong to $\iota(\lambda)$, then for some point $p \in \gamma$, the inequality $\max _{i=1,2} d\left(p, \partial_{i} W\right)<\delta_{1} / 3$ holds.

Compare this lemma with [5, Lemma 4.2].

Proof It follows from Lemma 3.1 that for $C_{1}$ large enough, the pleating map $\iota$ is $L$-bilipschitz for some universal constant $L>1$. Observe that $\iota\left(\mathbb{H}^{2}\right) \subset W$. Moreover, there is a constant $M_{0}>0$, that depends only on $L$, such that for every $p \in W$ we have $d\left(p, \iota\left(\mathbb{H}^{2}\right)\right)<M_{0}$

We choose $\delta_{1}>0$ as follows. Let $P_{0}$ be the pleated surface in $\mathbb{H}^{3}$ that has a single bending line $\gamma_{0}$, and with the bending angle equal to $\pi / 4$. Then $P_{0}$ is bounded by a quasicircle at $\partial \mathbb{H}^{3}$. Denote by $W_{0}$ the convex hull of this quasicircle and let $\partial_{i}\left(W_{0}\right)$, $i=1,2$, denote the two boundary components of $W_{0}$. Then there exists $\delta_{1}>0$ such that for every point $p \in \gamma_{0}$, we have $\max _{i=1,2} d\left(p, \partial_{i} W_{0}\right)>2 \delta_{1}$. Observe that $\gamma_{0}$ belongs to exactly one of the convex hull boundaries $\partial_{1} W_{0}$ and $\partial_{2} W_{0}$, so one of the numbers $d\left(p, \partial_{1} W_{0}\right)$ and $d\left(p, \partial_{2} W_{0}\right)$ is zero and the other one is larger than $2 \delta_{1}$. 
Assume that the first statement of the lemma is false. Then there exists a sequence of measured laminations $\left(\lambda_{n}, \mu_{n}\right)$ with the property $\mathcal{K}\left(\lambda_{n}\right) \rightarrow \infty$, and there are geodesics $l_{n} \in \lambda_{n}$, and points $p_{n} \in \gamma_{n}=\iota_{n}\left(l_{n}\right)$, such that the inequality

$$
\max _{i=1,2} d\left(p_{n}, \partial_{i} W_{n}\right) \leq \delta_{1}
$$

holds. We may assume that $p_{n}=p$, and $\gamma_{n}=\gamma$, for every $n$, where $p$ and $\gamma$ are fixed. Since $\iota_{n}$ is $L$-bilipschitz, after passing to a subsequence if necessary, the sequence $\iota_{n}$ converges (uniformly on compact sets) to a pleating map $\iota_{\infty}$. The pleating map $\iota_{\infty}$ corresponds to the pleating surface $P_{\infty}$, that has a single bending line $\gamma_{\infty}$, with the bending angle at least $\pi / 4$. Then $W_{n}$ converges to $W_{\infty}$ uniformly on compact sets in $\mathbb{H}^{3}$, where $W_{\infty}$ is the convex hull of the quasicircle that bounds $P_{\infty}$. It follows that $d\left(p_{n}, \partial_{i} W_{n}\right) \rightarrow d\left(p, \partial_{i} W_{\infty}\right)$. We may assume that $\gamma_{\infty}=\gamma_{0}$, where $\gamma_{0}$ is the bending line of the pleated surface $P_{0}$ defined above. Then we have $\max _{i=1,2} d\left(p, \partial_{i} W_{\infty}\right) \geq$ $\max _{i=1,2} d\left(p, \partial_{i} W_{0}\right)>2 \delta_{1}$. But this contradicts (4).

We now prove the second statement of the lemma. Let $\gamma$ be a geodesic in $W$ that is not in $\iota(\lambda)$. Then we can find a point $p \in \gamma$, such that $d(p, \iota(\lambda))>\mathcal{K}(\lambda)$. Assuming that the second statement is false, we again produce a sequence $\lambda_{n}$ with $\mathcal{K}\left(\lambda_{n}\right) \rightarrow \infty$, and such that for some sequence of geodesics $\gamma_{n} \subset W_{n}$, that do not belong to $\iota\left(\lambda_{n}\right)$, and all the points $p \in \gamma_{n}$, the inequality

$$
\max _{i=1,2} d\left(p, \partial_{i} W_{n}\right) \geq \delta_{1} / 3
$$

holds for $n$ large enough. By the previous discussion, there exists a sequence of points $p_{n} \in \gamma_{n}$, such that $d\left(p_{n}, \iota_{n}\left(\lambda_{n}\right)\right)>\mathcal{K}\left(\lambda_{n}\right)$.

Let $q_{n} \in \iota_{n}\left(\mathbb{H}^{2}\right)$ be points such that $d\left(p_{n}, q_{n}\right)<M_{0}$, where $M_{0}$ is the constant defined at the beginning of the proof. Let $z_{n} \in \mathbb{H}^{2}$, such that $q_{n}=\iota\left(z_{n}\right)$. We may assume that $z_{n}=i \in \mathbb{H}^{2}$ and $q_{n}=q$, for some point $q$ that we fix. Then $p_{n} \rightarrow p$, where $d(p, q) \leq M_{0}$. Moreover, since $\mathcal{K}\left(\lambda_{n}\right) \rightarrow \infty$, the pleating maps $\iota\left(\lambda_{n}\right)$ converge to an isometry uniformly on compact sets in $\mathbb{H}^{2}$. In particular, the sequence of convex hulls $W_{n}$ converges to a geodesic plane uniformly on compact sets, and therefore $d\left(p_{n}, \partial_{i} W_{n}\right) \rightarrow 0$. But this contradicts (5), and thus we have completed the proof of the lemma.

\section{$3.4(\epsilon, R)$ Skew pants}

We let $S$ continue to denote a closed surface with a generalized pants decomposition $\mathcal{C}$, and we fix a holomorphic representations $\rho_{z, w}$ as in Proposition 3.1. 
Let $\mathcal{C}_{0} \subset \mathcal{C}$ denote a subcollection of curves, and for each $C \in \mathcal{C}_{0}$ we choose a number $-(3 / 4) \pi<\theta_{C}<(3 / 4) \pi$ (for each curve $C \in\left(\mathcal{C} \backslash \mathcal{C}_{0}\right)$ we set $\left.\theta_{C}=0\right)$.

For $C \in \mathcal{C}$, let $\zeta_{C}, \eta_{C} \in \mathbb{D}$, where $\mathbb{D}$ denotes the unit disc in the complex plane. Let $\tau \in \mathbb{D}$ denote a complex parameter and let $t \in\{0,1\}$. Fix $R>1$, and let $z: \mathbb{D} \rightarrow \mathbb{C}_{+}^{\mathcal{C}}$ and $w: \mathbb{D} \rightarrow \mathbb{C}^{\mathcal{C}}$ be the mappings given by

$$
\begin{aligned}
z(C)(\tau) & =\frac{R}{2}+\frac{\tau \zeta_{C}}{2}, \\
w(C)(\tau, t) & =1+i t \theta_{C}+\frac{\tau \eta_{C}}{R} .
\end{aligned}
$$

The maps $z(\tau)$ and $w(\tau, t)$ are complex linear, and therefore holomorphic in $\tau$ and $t$. Therefore the induced family of representations $\rho_{\tau, t}=\rho_{z(\tau), w(\tau, t)}$ is holomorphic in $\tau$ and $t$. Note that $\rho_{\tau, t}$ depends on $R, \zeta_{C}, \eta_{C}$ and $\theta_{C}$, but we suppress this.

The representation $\rho_{0,0}$ is Fuchsian. Let $S_{0}$ denote the Riemann surface isomorphic to the quotient $\mathbb{H}^{2} / \rho_{0,0}\left(\pi_{1}(S)\right.$ ) (we also equip $S_{0}$ with the corresponding hyperbolic metric). Let $\mathcal{K}\left(\rho_{0,0}\right)$ denote the largest number so that the collection of collars (of width $\left.\mathcal{K}\left(\rho_{0,0}\right)\right)$ around the curves from $\mathcal{C}_{0}$ is disjoint on $S_{0}$.

The representation $\rho_{0,1}$ is not Fuchsian (unless $\theta\left(\mathcal{C}_{0}\right)=0$ ), and the following theorem gives a sufficient condition for it to be quasifuchsian.

We adopt the following notation. Let $G(\tau, t)=\rho_{\tau, t}\left(\pi_{1}(S)\right)$. If $G(\tau, t)$ is a quasifuchsian group we let $f_{\tau, t}: \partial \mathbb{H}^{2} \rightarrow \partial \mathbb{H}^{3}$, denote the quasiconformal map that conjugates $G(0,0)$ to $G(\tau, t)$. The following theorem is a generalization of [3, Theorem 2.2] (see Theorem 3.4 below). Assuming the above notation, we have:

Theorem 3.2 There exist universal constants $\hat{R}, \hat{\epsilon}, M>0$, such that the following holds. If $\mathcal{K}\left(\rho_{0,0}\right)>M$, then for every $R \geq \hat{R}$ and $|\tau|<\hat{\epsilon}$, and any choice of constants $\eta_{C}, \zeta_{C} \in \mathbb{D}$, and $-(3 / 4) \pi<\theta_{C}<(3 / 4) \pi$, for $C \in \mathcal{C}_{0}$, the group $G(\tau, 1)$ is quasifuchsian and the induced quasiconformal map $f_{\tau, 1} \circ\left(f_{0,1}\right)^{-1}$ (that conjugates $G(0,1)$ to $G(\tau, 1))$, is $K(\tau)$-quasiconformal, where

$$
K(\tau)=\frac{\widehat{\epsilon}+|\tau|}{\widehat{\epsilon}-|\tau|} .
$$

Let $\mathcal{C}_{0}(\tau, t)$ denote the collection of axes of elements of the form $\rho_{\tau, t}(c)$, where $c \in \pi_{1}(S)$ and $c$ belongs to the conjugacy class of some curve $C \in \mathcal{C}_{0}$. Then by definition, the set $\mathcal{C}_{0}(\tau, t)$ is invariant under the group $G(\tau, 1)$. Next, we prove that $\mathcal{C}_{0}(\tau, 1)$ is invariant under any Möbius transformation from $\operatorname{PSL}(2, \mathbb{C})$ that preserves the limit set of $G(\tau, 1)$. The following theorem is the main result of this section. 
Theorem 3.3 There exist constants $\hat{\epsilon}_{1}, M_{1}>0$, with the following properties. Assume that $\mathcal{K}\left(\rho_{0,0}\right)>M_{1}$ and let $|\tau|<\hat{\epsilon}_{1}$. If $T \in \operatorname{PSL}(2, \mathbb{C})$, is a Möbius transformation that preserves the limit set of $G(\tau, 1)$, then the set of geodesics $\mathcal{C}_{0}(\tau, 1)$ is invariant under $T$.

Compare this theorem with [5, Lemma 4.2].

Proof Let $W(\tau, t)$ denote the convex hull of the limit set of $G(\tau, t)$. It follows from Lemma 3.3 that for $\mathcal{K}\left(\rho_{0,0}\right)$ large enough, the following holds:

(1) For every $\gamma \in \mathcal{C}_{0}(0,1)$ and $p \in \gamma$, the inequality

$$
\max _{i=1,2} d\left(p, \partial_{i} W(0, t)\right)>\delta_{1}
$$

holds.

(2) For every $\gamma \subset W(0,1)$, there exists $p \in \gamma$ such that

$$
\max _{i=1,2} d\left(p, \partial_{i} W(0,1)\right)<\frac{\delta_{1}}{2} \text {. }
$$

Then by Theorem 3.2 we can choose $\hat{\epsilon}_{1}$ small enough so that for $|\tau|<\hat{\epsilon}_{1}$, the constant $K(\tau)$ (from Theorem 3.2) is close enough to 1 , so that the following holds:

(1) For every $\gamma \in \mathcal{C}_{0}(\tau, 1)$ and $p \in \gamma$, the inequality

$$
\max _{i=1,2} d\left(p, \partial_{i} W(0, t)\right)>\frac{4 \delta_{1}}{5}
$$

holds,

(2) For every $\gamma \subset W(0,1)$, there exists $p \in \gamma$ such that

$$
\max _{i=1,2} d\left(p, \partial_{i} W(0,1)\right)<\frac{2 \delta_{1}}{3} .
$$

Then any Möbius transformation $A \in \operatorname{PSL}(2, \mathbb{C})$ that preserves $W(\tau, 1)$ will also preserve the set $\mathcal{C}(\tau, 1)$. This proves the theorem.

\subsection{A proof of Theorem 3.2}

We need to prove that $G(\tau, 1)$ is a quasifuchsian group. The last estimate in Theorem 3.2 then follows from the fact that a holomorphic map from the unit disc into the Teichmüller space of a Riemann surface is a contraction with respect to the hyperbolic metric on the unit disc and the Teichmüller metric. 
Theorem 3.4 [3, Theorem 2.2] There exist universal constants $\hat{R}, \hat{\epsilon}$, such that the following holds. For every $R \geq \widehat{R}$ and $|\tau|<\hat{\epsilon}$, and any choice of constants $\eta_{C}, \zeta_{C} \in \mathbb{D}$, the group $G(\tau, 0)$ is quasifuchsian, and the induced quasiconformal map $f_{\tau, 0}$ that conjugates $G(0,0)$ to $G(\tau, 0)$, is $K(\tau)$-quasiconformal, where

$$
K(\tau)=\frac{\widehat{\epsilon}+|\tau|}{\widehat{\epsilon}-|\tau|} .
$$

The group $G(\tau, 1)$ is obtained from the group $G(\tau, 0)$, by bending along the lifts of curves $C \in \mathcal{C}_{0}$, for the angle $\theta_{C}$. It follows from Theorem 3.1 that the group $G(\tau, 1)$ is quasifuchsian if $\mathcal{K}\left(\rho_{0,0}\right)>C$, and if the map $f_{\tau, 0}$ is $K$-quasiconformal, where $K$ is close enough to 1 . But it follows from Theorem 3.4 that for $|\tau|$ small enough this will be the case. This proves Theorem 3.2.

\section{The lower bound}

\subsection{Amalgamating two representations}

Let $S$ denote a closed surfaces with generalized pants decompositions $\mathcal{C}$, and let $\rho: \pi_{1}(S) \rightarrow \operatorname{PSL}(2, \mathbb{C})$ denote an admissible (in sense of Definition 3.1) representation with the reduced Fenchel-Nielsen coordinates satisfying the inequalities

$$
\begin{aligned}
\left|\mathbf{h l}(C)-\frac{R}{2}\right| & \leq \epsilon, \\
|s(C)-1| & \leq \frac{\epsilon}{R},
\end{aligned}
$$

for some $\epsilon, R>0$, and $C \in \mathcal{C}$. We say that such a representation is $(\epsilon, R)$-good.

Let $\mathbf{M}^{3}$ denote a closed hyperbolic manifold such that $\mathbf{M}^{3}=\mathbb{H}^{3} / \Gamma$ for some Kleinian group $\Gamma$. In [3] we proved that one can find many $(\epsilon, R)-\operatorname{good}$ representations $\rho: \pi_{1}(S) \rightarrow \Gamma$, for a given $\epsilon>0$ and $R$ large enough. Moreover, if $A \in \Gamma$ has the translation length $\mathbf{I}(A)$ satisfying the inequality $|\mathbf{I}(A)-R| \leq \epsilon / 2$, then we can find such $\rho$ so that $A$ is in the image of $\rho$. From now on we assume that such $A \in \Gamma$ is primitive, that is $A$ is not equal to an integer power of another element of $\Gamma$.

In particular, it follows from [3, Section 4] (the statements about the equidistribution of $(\epsilon, R)$-good pairs of skew pants around a given closed curve in $\mathbf{M}^{3}$ whose length is $\epsilon$ close to $R)$ that we can find two $(\epsilon, R)$-good representations $\rho(i): \pi_{1}(S(i)) \rightarrow \Gamma$, $i=1,2$, where $S(1)$ and $S(2)$ are two closed surfaces with pants decompositions $\mathcal{C}(i)$, and two pairs of pants $\Pi_{i}^{+}$and $\Pi_{i}^{-}$with the following properties: 
- There are two oriented, degree one curves $C(i) \in \mathcal{C}(i)$, and $c(i) \in \pi_{1}(S(i))$ in the conjugacy classes of $C(1)$ and $C(2)$ respectively, such that $\rho(1)(C(1))=$ $\rho(2)(C(2))=[A]$, where $[A]$ is the conjugacy class of a given primitive element $A \in \Gamma$, whose translation length $\mathbf{I}(A)$ satisfies the inequality $|\mathbf{I}(A)-R| \leq \epsilon / 2$.

- Let $\gamma$ denote the closed geodesic corresponding to $A$. There exist two pars of skew pants $\Pi_{i}^{+}$and $\Pi_{i}^{-}$in $\rho(i)\left(\pi_{1}(S(i))\right)$ such that $\gamma$ is positively oriented boundary component of $\Pi_{i}^{+}$and negatively oriented for $\Pi_{i}^{-}$, and recalling the notation from [3] we have the inequality

$$
\left|\operatorname{foot}_{\gamma}\left(\Pi_{2}^{+}\right)-\operatorname{foot}_{\gamma}\left(\Pi_{1}^{-}\right)-\frac{\pi}{2}\right| \leq \frac{\epsilon}{R} .
$$

After replacing $S(1)$ and $S(2)$ with appropriate finite degree covers if necessary, we may assume in addition to the above two conditions the following also hold:

- The curves $C(1)$ and $C(2)$ are nonseparating simple closed curves in $S(1)$ and $S(2)$ respectively.

- The surfaces $S(1)$ and $S(2)$ have the same genus.

- By Proposition 3.1 the representation $\rho(i)$ can be embedded in the holomorphic family of representations $\rho_{\tau, t}(i)$. We may assume that $\mathcal{K}\left(\rho_{0,0}(S(i))\right)>C_{1}$, $i=1,2$, where $C_{1}$ is the constant from Theorem 3.3.

We now fix such two representations $\rho(1)$ and $\rho(2)$, surfaces $S(1)$ and $S(2)$, and the two oriented curves $C(1)$ and $C(2)$ (we also fix the corresponding primitive element $A \in \Gamma)$.

Let $i \in\{1,2\}$. For $n>1$, let $S_{n}(1)$ and $S_{n}(2)$ denote two primitive degree $n$ covers of $S(1)$ and $S(2)$ respectively (a finite cover of a surface is primitive if it does not factor through an intermediate cover), such that for some $1 \leq k \leq(n-1)$, the curves $C(1)$ and $C(2)$ have two degree $k$ lifts $C_{n}(1)$ and $C_{n}(2)$. Then $C_{n}(1)$ and $C_{n}(2)$ are two oriented, nonseparating simple closed curves in $S_{n}(1)$ and $S_{n}(2)$ respectively. We then have the two induced representations $\rho_{n}(i): \pi_{1}\left(S_{n}(i)\right) \rightarrow \Gamma$, that also satisfy the above five conditions, except that

$$
\rho_{n}(1)\left(\pi_{1}\left(S_{n}(1)\right)\right) \cap \rho_{n}(2)\left(\pi_{1}\left(S_{n}(2)\right)\right)=\left\{A^{k}\right\} .
$$

We amalgamate them as follows. Cut the surface $S_{n}(i)$ along $C_{n}(i)$, to get two topological surfaces $\bar{S}_{n}(i), i=1,2$, each having two boundary components $C_{n}^{1}(i)$ and $C_{n}^{2}(i)$. We glue together the surfaces $\bar{S}_{n}(1)$ and $\bar{S}_{n}(2)$ by gluing $C_{n}^{j}(1)$ to $C_{n}^{j}(2)$, $j=1,2$, and obtain a closed topological surface $S_{n}$ (this is well defined up to a twist by $\Re(\mathbf{l}(A))$ which has a period $k)$. The surface $S_{n}$ has the induced generalized pants 
decomposition $\mathcal{C}_{n}$. The pair of curves $C_{n}^{1}(1)$ and $C_{n}^{1}(2)$ that were glued together produce a closed curve $C_{n}^{1}$ in $S_{n}$. Similarly, the pair of curves $C_{n}^{2}(1)$ and $C_{n}^{2}(2)$ that were glued together produce a closed curve $C_{n}^{2}$ in $S_{n}$. We set $\mathcal{C}_{0, n}=\left\{C_{n}^{1}, C_{n}^{2}\right\}$.

Then there is the induced representation $\rho_{n}: \pi_{1}\left(S_{n}\right) \rightarrow \Gamma$. We orient the curves $C_{n}^{1}$ and $C_{n}^{2}$ such that for any choice of $c_{i} \in \pi_{1}\left(S_{n}\right)$, where $c_{i}$ is in the conjugacy class of $C_{n}^{i}$, we have that both $\rho_{n}\left(c_{1}\right)$ and $\rho_{n}\left(c_{2}\right)$ are in the conjugacy class of $A^{k}$ in $\Gamma$.

The representation $\rho_{n}$ has the reduced Fenchel-Nielsen coordinates satisfying the relations

$$
\begin{aligned}
\left|\mathbf{h l}(C)-\frac{R}{2}\right| & \leq \epsilon, \\
|s(C)-1| & \leq \frac{\epsilon}{R},
\end{aligned}
$$

if $C$ does not belong to $\mathcal{C}_{0, n}$, and

$$
\left|s(C)-\left(1+i \frac{\pi}{2}\right)\right| \leq \frac{\epsilon}{R},
$$

if $C \in \mathcal{C}_{0, n}$.

It follows from Theorem 3.2 that for $\epsilon$ small enough and $R$ large enough, the group $\rho_{n}\left(\pi_{1}\left(S_{n}\right)\right)$ is quasifuchsian. In the remainder of this subsection we prove that the group $\rho_{n}\left(\pi_{1}\left(S_{n}\right)\right)$ is a maximal subgroup of $\Gamma$.

First we prove a preliminary lemma. Let $\bar{S}$ be a surface with boundary components $C_{+}$ and $C_{-}$, oriented so that $\bar{S}$ is on the left of $C_{+}$and on the right of $C_{-}$. We say that $f: \bar{S} \rightarrow \mathbf{M}^{3}$ is rejoinable if the restrictions of $f$ to $C^{+}$and $C_{-}$respectively are freely homotopic in $\mathbf{M}^{3}$. We say $(f, \bar{S})$ is geodesically rejoinable if $\left.f\right|_{C_{+}}$and $\left.f\right|_{C_{-}}$ map to the same closed geodesic in $\mathbf{M}^{3}$. In this case we say a rejoining of $(f, \bar{S})$ is a homeomorphism $h: C_{+} \rightarrow C_{-}$such that $f \circ h=f$, and we say $(f, \bar{S} / h)$ is $\bar{S}$ rejoined by $h$.

Lemma 4.1 If $(f, \bar{S})$, and $(g, \bar{T})$ are (geodesically) rejoinable surfaces, and $\pi: \bar{S} \rightarrow \bar{T}$ is a finite cover such that $g \circ \pi$ is homotopic to $f$, then for any rejoining $h$ of $(f, \bar{S})$ we can find a rejoining $k$ of $(g, \bar{T})$ such that $(f, \bar{S})$ rejoined by $h$ covers $(g, \bar{T})$ rejoined by $k$.

Proof Left to the reader.

The following theorem is a corollary of Theorem 3.3. We adopt the following definition. Let $f: S \rightarrow \mathbf{M}^{3}$ be a map such that $f(S)$ is a quasifuchsian surface in $\mathbf{M}^{3}$, and 
let $\mathcal{C}_{0}$ denote a collection of disjoint simple closed curves on $S$. We say that $f$ is bent along each curve of $\mathcal{C}_{0}$ and nearly locally isometric on $S \backslash \mathcal{C}_{0}$ if the induced map $f_{*}: \pi_{1}(S) \rightarrow \Gamma$ is of the form $\rho_{\tau, 1}$ for some $|\tau| \leq \hat{\epsilon}$.

Theorem 4.1 Let $S$ be a closed surface. Suppose that $f: S \rightarrow \mathbf{M}^{3}$ is a $\pi_{1}$-injective and quasifuchsian, and $\mathcal{C}_{0}$ is a collection of disjoint simple closed curves on $S$, such that $f$ is bent along each curve of $\mathcal{C}_{0}$ and nearly locally isometric on $S \backslash \mathcal{C}_{0}$. Suppose that $f=g \circ \pi$, where $\pi: S \rightarrow Q$ is a covering, and $g: Q \rightarrow \mathbf{M}^{3}$ is $\pi_{1}$-injective and quasifuchsian. Then we can find a collection of simple closed curves $\hat{\mathcal{C}}_{0}$ on $Q$ such that $\mathcal{C}_{0}=\pi^{-1}\left(\widehat{\mathcal{C}_{0}}\right)$.

Proof We get a discrete lamination $\widetilde{\mathcal{C}}_{0}$ on $\mathbb{H}^{2}$, which we push forward by $\tilde{f}=\widetilde{g}$ to $\mathbb{H}^{3}$. We find a homomorphism $\sigma: \operatorname{Deck}\left(\mathbb{H}^{2} / Q\right) \rightarrow \Gamma$ such that $\tilde{f}(\gamma(x))=\sigma(\gamma)(\tilde{f}(x))$ for every $x \in \mathbb{H}^{2}$ and $\gamma \in \operatorname{Deck}\left(\mathbb{H}^{2} / Q\right)$.

We let $G=\sigma\left(\operatorname{Deck}\left(\mathbb{H}^{2} / Q\right)\right)$, and $H=\sigma\left(\operatorname{Deck}\left(\mathbb{H}^{2} / S\right)\right)<G$. Then $[G: H]<\infty$, and $G$ and $H$ are quasifuchsian groups, and they have the same limit set, so by Theorem 3.3 every element of $G$ maps $\widetilde{g}\left(\widetilde{\mathcal{C}}_{0}\right)$ to itself. Hence $\operatorname{Deck}\left(\mathbb{H}^{2} / Q\right)$ maps $\widetilde{\mathcal{C}}_{0}$ to itself, so $\widetilde{\mathcal{C}_{0}}$ is a lift of $\hat{\mathcal{C}}_{0}$ on $Q$, and hence $\mathcal{C}_{0}$ is.

Theorem 4.2 The quasifuchsian group $\rho_{n}\left(\pi_{1}\left(S_{n}\right)\right)<\Gamma$ is a maximal surface subgroup of $\Gamma$, that is, if $\rho_{n}\left(\pi_{1}\left(S_{n}\right)\right)<G$ for a surface subgroup $G<\Gamma$, then $G=$ $\rho_{n}\left(\pi_{1}\left(S_{n}\right)\right)$.

Proof For simplicity let $G_{n}=\rho_{n}\left(\pi_{1}\left(S_{n}\right)\right)$ and $G(1)=\rho(1)\left(\pi_{1}(S(1))\right)$. Also set $G_{n}(1)=\rho_{n}\left(\pi_{1}\left(\bar{S}_{n}(1)\right)\right)$, where we consider $\pi_{1}\left(\bar{S}_{n}(1)\right)$ as a subgroup of $\pi_{1}\left(S_{n}\right)$.

Let $f_{n}: S_{n} \rightarrow \mathbf{M}^{3}$ denote the continuous map that corresponds to the representation $\rho_{n}$. We claim that $f_{n}: S_{n} \rightarrow \mathbf{M}^{3}$ is primitive. If not, we can find a Riemann surface $Q$ and $\pi: S_{n} \rightarrow Q$ and $g: Q \rightarrow \mathbf{M}^{3}$ such that $g \circ \pi=f_{n}$ and $d>1$ where $d$ is the degree of the cover $\pi$. We recall that $f_{n}$ is bent along $C_{n}^{1}$ and $C_{n}^{2}$, and nearly isometric on the complement. So by Theorem $4.1,\left\{C_{n}^{1}, C_{n}^{2}\right\}$ are the lifts by $\pi$ of some set $\mathcal{C}_{Q}$ of simple closed curves on $Q$. So $\left|\mathcal{C}_{Q}\right|=1$ or $\left|\mathcal{C}_{Q}\right|=2$.

If $\left|\mathcal{C}_{Q}\right|=2$, then each component of $S_{n} \backslash \cup C_{n}^{i}$ maps by degree $d$ to a component of $Q \backslash \mathcal{C}_{Q}$. We can then write $Q \backslash \mathcal{C}_{Q}=\bar{Q}(1) \cup \bar{Q}(2)$ such that $\pi: \bar{S}_{n}(i) \rightarrow \bar{Q}(i)$ is a degree $d$ cover, and then by Lemma 4.1 we can rejoin the boundary curves of $\bar{Q}(1)$ to form $Q^{\prime}(1)$ such that $S_{n}(1)$ is a cover of $Q^{\prime}(1)$. But then we get a subgroup $G_{Q^{\prime}}$ of $G_{n}(1)\left(G_{Q^{\prime}}=\pi_{1}\left(Q^{\prime}(1)\right)\right)$, and $G_{n}(1)<G_{Q^{\prime}} \cap G(1)<G(1)$, where both inclusions are proper. The first inclusion is proper because $A^{k / d} \in G_{Q^{\prime}} \cap G(1) \backslash G_{n}(1)$, and the 
second is proper because $k<n$. This contradicts the assumption on the maximality of $G_{n}(1)$.

If $\left|\mathcal{C}_{Q}\right|=1$, we let $\mathcal{C}_{Q}=\left\{C_{Q}\right\}$. First suppose that $C_{Q}$ is nonseparating. Then writing $Q \backslash C_{Q}=\bar{Q}$ we find that $\bar{S}_{n}(1)$ and $\bar{S}_{n}(2)$ are both degree $d / 2$ covers of $\bar{Q}$. But then we can reassemble $\bar{Q}$ to make $Q^{\prime}$ (by Lemma 4.1) such that $S_{n}(1)$ is a degree $d / 2$ cover of $Q^{\prime}$, when $d / 2 \leq k$. Then we arrive at a contradiction by the same reasoning as before.

Finally, suppose that $C_{Q}$ is separating. Then we can write $Q \backslash C_{Q}=\bar{Q}(1) \cup \bar{Q}(2)$ so that the restriction of $\pi$ to $\bar{S}_{n}(i)$ is a cover of $\bar{Q}(i)$. Then the conjugacy classes for $C_{n}^{1}$ and $C_{n}^{2}$, oriented as curves covered by the axis of $A$, are both in $\left[A^{k}\right]$, but $C_{n}^{1}$ and $C_{n}^{2}$ both cover $C_{Q}$ with opposite orientations, so the conjugacy class for $C_{Q}$ must be both $\left[A^{l}\right]$ and $\left[A^{-l}\right]$, where $l=2 k / d$. But then $B^{-1} A^{l} B=A^{-l}$ for some $B \in \Gamma$, which means that $B$ preserves the axis of $A$ and reverses its orientation; such $B$ would have a fixed point in $\mathbb{H}^{3}$, which is a contradiction.

\subsection{The lower bound}

We now proceed to prove the lower bound

$$
\left(c_{1} g\right)^{2 g} \leq s_{1}\left(\mathbf{M}^{3}, g\right),
$$

for $g$ large enough, from Theorem 1.1.

By the above theorem the representation $\rho_{n}: \pi_{1}\left(S_{n}\right) \rightarrow \Gamma$, is maximal. It remains to count the number of such representations. Let $g_{n}$ denote the genus of the surface $S_{n}$. If $g_{0}$ denotes the genus of the surfaces $S(1)$ and $S(2)$, we have

$$
g_{n}=n\left(2 g_{0}-1\right) \text {. }
$$

Given a closed surface $S_{0}$, Let $m_{n}\left(S_{0}\right)$ denote the number of maximal degree $n$ covers of $S_{0}$. Let $C_{0}$ denote a simple closed and nonseparating curve in $S_{0}$. For $1 \leq k \leq n$, by $m_{n}\left(S_{0}, C_{0}, k\right)$ we denote the number of maximal $n$ degree covers of $S_{0}$ such that the curve $C_{0}$ has at least one lift of degree $k$. Clearly the number $m_{n}\left(S_{0}, C_{0}, k\right)$ does not depend on the choice of the simple closed and nonseparating curve $C_{0}$, so we sometimes write $m_{n}\left(S_{0}, k\right)=m_{n}\left(S_{0}, C_{0}, k\right)$.

Theorem 4.3 Let $g_{0}$ denote the genus of $S_{0}$. Then for $n$ large we have

$$
m_{n}\left(S_{0}\right)=(n !)^{g_{0}-2}(1+o(1)),
$$

where $o(1) \rightarrow 0$ when $n \rightarrow \infty$. Moreover, for some $1 \leq k \leq(n-1), k=k\left(n, g_{0}\right)$, we have

$$
m_{n}\left(S_{0}, k\right)>((n-1) !)^{g_{0}-2}(1+o(1)) .
$$


Proof The first equality directly follows from Corollary 3 and the formula in Section 4.4 in [6], which shows that a random finite cover of a closed surface is maximal. It remains to prove the second inequality.

Since

$$
\sum_{k=1}^{n} m_{n}\left(S_{0}, k\right) \geq m_{n}\left(S_{0}\right),
$$

it follows that for some $1 \leq k \leq n$, the second inequality in the statement of the theorem holds. The following lemma implies that this inequality holds for some $1 \leq k \leq(n-1)$.

Lemma 4.2 The inequality $m_{n}\left(S_{0}, 1\right) \geq m_{n}\left(S_{0}, n\right)$, holds for every $n$.

Proof Let $C_{0}$ and $D_{0}$ be two simple closed and nonseparating curves on $S_{0}$, that intersect exactly once. Let $S_{n}$ be a degree $n$ cover of $S_{0}$, such that the curve $C_{0}$ has a degree $n$ lift which we denote by $C_{n}$. Then $C_{n}$ is the only lift of $C_{0}$. We show that in this case, every lift of the curve $D_{0}$ is a degree one lift. Let $\widetilde{S}_{0}=S_{0} \backslash C_{0}$ and $\widetilde{S}_{n}=S_{n} \backslash C_{n}$, denote the two surfaces each having exactly two boundary components. Then $\widetilde{S}_{n}$ covers $\widetilde{S}_{0}$, because $C_{n}$ is the only lift of $C_{0}$ to $S_{n}$. After removing the curve $C_{0}$ from $S_{0}$, the closed curve $D_{0}$ becomes an interval $I_{0} \subset \widetilde{S}_{0}$, whose endpoints lie on different boundary components of $\widetilde{S}_{0}$. Therefore, every lift of $I_{0}$ to $\widetilde{S}_{n}$ is a degree one lift. This proves the statement.

Restricting to when $S_{n}$ is a maximal cover yields the inequality $m_{n}\left(S_{0}, C_{0}, n\right) \leq$ $m_{n}\left(S_{0}, D_{0}, 1\right)$. Since $m_{n}\left(S_{0}, C_{0}, k\right)=m_{n}\left(S_{0}, D_{0}, k\right)=m_{n}\left(S_{0}, k\right)$, it follows that $m_{n}\left(S_{0}, 1\right) \geq m_{n}\left(S_{0}, n\right)$, and we have proved the lemma.

This proves the theorem.

Now fix a large $n$ and choose $1 \leq k \leq(n-1)$ so that the second inequality in Theorem 4.3 holds. We then amalgamate any two maximal covers $S_{n}(1)$ and $S_{n}(2)$ along the curves $C_{n}(1)$ and $C_{n}(2)$ that are both $k$ degree lifts of the curves $C(1)$ and $C(2)$ respectively (there may be more than one such $k$ degree lift, but we choose arbitrarily). Then the corresponding group $\rho_{n}\left(\pi_{1}\left(S_{n}\right)\right)<\Gamma$ is maximal surface subgroup of $\Gamma$. Combining the above formula for $g_{n}$ with the Theorem 4.3, we derive the estimate (7) for some $c_{1}>0$.

\section{References}

[1] E A Bender, E R Canfield, The asymptotic number of rooted maps on a surface, J. Combin. Theory Ser. A 43 (1986) 244-257 MR867650 
[2] A Douady, C J Earle, Conformally natural extension of homeomorphisms of the circle, Acta Math. 157 (1986) 23-48 MR857678

[3] J Kahn, V Markovic, Immersing almost geodesic surfaces in a closed hyperbolic three manifold arXiv:0910.5501

[4] C Kourouniotis, Complex length coordinates for quasi-Fuchsian groups, Mathematika 41 (1994) 173-188 MR1288062

[5] J D Masters, Counting immersed surfaces in hyperbolic 3-manifolds, Algebr. Geom. Topol. 5 (2005) 835-864 MR2153105

[6] T W Müller, J-C Puchta, Character theory of symmetric groups and subgroup growth of surface groups, J. London Math. Soc. 66 (2002) 623-640 MR1934296

[7] C Series, An extension of Wolpert's derivative formula, Pacific J. Math. 197 (2001) 223-239 MR1810217

[8] S P Tan, Complex Fenchel-Nielsen coordinates for quasi-Fuchsian structures, Internat. J. Math. 5 (1994) 239-251 MR1266284

Department of Mathematics, Brown University

Providence RI 02912, USA

Department of Mathematics, California Institute of Technology

Pasadena CA 91105, USA

kahn@math.brown.edu, markovic@caltech.edu

http://www.math.brown.edu/ kahn/, http://www.its.caltech.edu/ markovic

Proposed: David Gabai

Seconded: Tom Mrowka, Walter Neumann
Received: 5 January 2011

Revised: 26 October 2011 